\title{
Chen D, Walther A, Moberg A, Jones P, Jacobeit J, Lister D | European trend atlas of extreme temperature and precipitation records
}

\author{
2015 | Springer Dordrecht, Heidelberg, New York, London | 178 pp | 159 figs, \\ 11 tables | Hardcover ISBN: 978-94-017-9311-7 USD 179.00, EUR 139.09 | E-book ISBN: \\ 978-94-017-9312-4 USD 139.00, EUR 107.09
}

\author{
Andreas Hoy $^{1} \cdot$ Stephanie Hänsel $^{1}$ \\ (c) Springer-Verlag Berlin Heidelberg 2015
}

Climate change is most visible and dangerous to society when it comes to extremes. Hence, an atlas that systematically illustrates the observed changes in temperature and precipitation extremes is strongly needed and desired. Thereby, the analysis of long-term time series is particularly attractive. This atlas advertises "an easy way to identify spatial patterns for a given time period, region, season, and index". Unfortunately, the hopes and expectations raised by the title and abstract are abated by the content and structure of the book.

The atlas builds on the results of the European Union's 5th Framework Programme project EMULATE (European and North Atlantic daily to multidecadal climate variability; 2002-2005) and more precisely on an internal report at the University of Gothenburg in 2006. The current book presents a subset of 27 daily temperature and precipitation indices (out of 64 EMULATE indices), calculated from 109 stations, to a wider audience. The trends for 1901-2000 are displayed in seasonal maps and time series are additionally plotted from longer records starting in 1801 and 1851. Although more recent years are easily accessible (e.g. http://eca.knmi.nl/), the authors have made no effort to update the time series to the present.

The book consists of five chapters and an appendix, which is predominantly figures and tables and a comparatively minor amount of text. The first chapter explains the motivation behind the book and briefly introduces the state of knowledge. Chapter 2 provides a short overview about

Stephanie Hänsel

stephanie.haensel@ioez.tu-freiberg.de

1 Interdisciplinary Environmental Research Center (IÖZ), TU Bergakademie Freiberg, Brennhausgasse 14, 09599 Freiberg, Germany the applied data and methods. In this respect, the nonclimatologist reader probably needs to consult the given references. Perhaps through oversight, essential information such as altitude and the maximum extent of the time series are missing in the table of the utilized meteorological stations.

The third chapter "Atlas of the Trend Analysis" forms the heart of the book. A meaningful figure numbering, correct figure captions and a well-structured figure list are lacking and would have helped the reader to navigate the book as it is intended to be used - as an atlas. Unfortunately, every page is assigned solely a figure number and each figure caption refers only to the upper left-hand illustration/graphic out of eight. Skimming through more than 1000 seasonal time series plots when searching for some specific index, season and station becomes quite time-consuming and is sub-standard for an atlas. Furthermore, the color-scale of the maps is sub-optimal and no attempts towards optimizing the time series plots are visible-these are likely the unedited output of the statistical software program "R". Such figures might be sufficient for an electronic supplement of an internal report, but not for a scientific book.

The final two chapters, "Summary of Estimated Trends" and "Summary and Conclusions", briefly summarize some information given by the extensive figures in chapter 3 . Nonetheless, our expectations of clear figures or tables emphasizing the main findings are not met by the presented tables on the fraction of positive and negative (statistically significant) trends and regional trend levels. The appendix contains some additional tables illustrating the trends of selected indices.

We hope that a future second edition of the atlas can fulfill the authors' claim that "the atlas clearly shows that the climate in Europe has changed over the last 100 to 150 years". 